\title{
Natural gas pipeline reliability analysis based on utility theory
}

\author{
Zhang Xinsheng \\ School of Management, \\ Xi'an University of Architecture \& Technology, \\ Xi'an Shaanxi 710055, China \\ xinsheng.zh@gmail.com \\ Li Wentao \\ School of Management, \\ Xi'an University of Architecture \& Technology, \\ Xi'an Shaanxi 710055, China \\ Lwtfl1225@126.com
}

\author{
Luo Zhengshan \\ School of Management, \\ Xi'an University of Architecture \& Technology, \\ Xi'an Shaanxi 710055, China line 4: e-mail: \\ luozhengshan@163.com \\ He Hongyan \\ School of Management, \\ Xi'an University of Architecture \& Technology, \\ Xi'an Shaanxi 710055, China \\ 799166584@qq.com
}

\begin{abstract}
China's natural gas pipelines construction began in the 1960s, As the service time increases, the corrosion cause the operation reliability decreases most of the current in-service pipelines are in the aging corrosion phase, safe and reliable operations of these pipelines are related to economic development and social stability. It is of great importance and practical significance to study when the corrosion pipelines will retire and how to guarantee these pipelines operating under safe and reliable condition. Firstly, combined with theory of the remaining strength and remaining life we analyze the corrosion leakage probability of pipeline remaining life using the exponential distribution; and then we calculate the internal economic loss value when the in-service pipelines are replaced ahead of scheduled time; Finally, e evaluate the economic loss, loss of life and damage to the environment in terms of the utility function to get the corresponding external loss value; and make sure the most optimal date to exchange the aging corrosion pipelines.
\end{abstract}

Keywords- Natural gas pipeline; aging corrosion phase; reliability; leakage probability; utility function

\section{INTRODUCTION}

China's natural gas pipeline start to construct from the 1960s, most of the existing pipelines has been close to or exceed the design lifetime, get into an accident-prone period. One main cause of the accidents is the pipe corrosion, and the direct cause of the position was due to the partial wall thinning caused by stress concentration. Because of natural gas pressure delivery, when part of the pipelines wall thinning to a certain extent, they will burst and cause leakage. Therefore the operation reliability of the older pipelines need to be studied[1].

According to the European, and the United States criteria for the classification of the severity of pipeline accidents, accidents generally not divided into three modes, leak、 perforation and rupture respectively[2]. Because pipeline corrosion failure is one of the most common problems, corrosion caused by leakage of objective factors is chosen as the study. Pipeline corrosion problems not only bring huge loss to the national economy, but also cause great risk to the safe operation[3].Especially, the older pipelines leak-prone due to corrosion problems, and once the concentration reaches a certain amount of leakage across the fire source and can cause a fire explosion easily. Moreover, it also causes property damage, personal injury and environmental pollution. With the development of society, people's attention to the environment, public safety and the necessity of the pipeline operation reliability is gradually increased. Therefore, the safety and reliability research of the older gas pipeline will have an important practical significance.

\section{RESIDUAL STRENGTH AND REMAINING LIFE}

Reliability evaluation of pipeline corrosion mainly involves evaluation of the residual strength and residual life prediction of the pipeline[4]. An important part of the residual strength of corroded pipeline assessment is to calculate the maximum size of the allowed defects of the pipeline, or to calculate maximum safe pressure of the pipeline in certain operating pressure[5-6].Research of residual strength of corroded pipeline and evaluation of the reliability of pipeline in service will have a great theoretical significance for the maintenance and replacement of piping systems serving[7]. In both corrosion and to meet the residual strength assessment conditions, prediction of the remaining lifetime of corroded pipeline is mainly analysis and prediction of development trend of pipeline corrosion and the effective time of reliable operation, using the $\mathrm{B} 31 \mathrm{G}$ criteria to evaluate residual strength of Corroded Pipeline generally.

\section{A. Residual Strength}

The B31G criterion (ASME 1993) is widely used to assess corroded pipelines. The main equations in the ASME B31G criteria (1993) can be summarized as follows. The maximum allowable design pressure in $\mathrm{B} 31 \mathrm{G}$ criterion is expressed as: 


$$
P=\frac{2 S M Y S}{D} \times F \times t
$$

Where:

$P$ — the maximum allowable design pressure;

SMYS — the Specified Minimum Yield Strength;

$F \_$the design factor, which is normally 0.72 ;

$t-$ wall-thickness of the pipe.

For the short of corrosion, corrosion region using the parabolic approximation, the maximum safe pressure $P^{\prime}$ according to the formula (2-2) calculation,

$$
P^{\prime}=1.1 P \times\left[\frac{1-\frac{2}{3} \times \frac{d}{t}}{1-\frac{2}{3}\left(\frac{d}{t \times \sqrt{A^{2}+1}}\right)}\right] P^{\prime} \leq P \quad A \leq 4
$$

For the long corrosion, corrosion region using the parabolic approximation, the maximum safe pressure $P^{\prime}$ according to the formula (2-3) calculation, i.e.,

$$
P^{\prime}=1.1 P \times\left[\frac{1-\frac{d}{t}}{1-\left(\frac{d}{t \times \sqrt{A^{2}+1}}\right)}\right] P^{\prime} \leq P \quad A \leq 4
$$

Where, $A=0.894\left(\frac{L}{\sqrt{D t}}\right)$

The Maximum Allowable Operating Pressure $(M A O P)$ is not more than the maximum allowable design pressure $P$ given by Equation (2-1) . Equating the Safe Maximum Pressure Level $P^{\prime}$ to the Maximum Allowable Operating Pressure (MAOP), the maximum allowable defect depth $d_{\text {allow }}$ is:

a) When corrosion is approximately parabolic shape, for $A \leq 4$,

$$
d_{\text {allow }}=\frac{3 t}{2}\left[\frac{1-M A O P / 1.1 P}{1-M A O P /\left(1.1 P \sqrt{A^{2}+1}\right)}\right]
$$

b) When corrosion is approximately rectangular shape, for $A>4$,

$$
d_{\text {allow }}=\left[1-\frac{M A O P}{1.1 P}\right] \times t
$$

\section{B. Remaining Life}

\section{1) The Basic Theory[8]}

After the pipeline corrosion, wall becomes thin. And ability of withstand the internal pressure is reduced, so pipeline leak and rupture resistance ability decrease. When the internal pressure is bigger than the limit of the carrying capacity of pipeline corrosion, pipeline will leak or be rupture. That is to say, pipeline current wall thickness thin $d$ to the allowed minimum wall thickness $d_{\text {min }}$, pipeline reach to its service life, the difference between the expected service life and the current service time is the remaining life. The mathematical model is:

$$
T_{r}=\sum_{i=1}^{n} \frac{d_{i}}{v_{i}}=\frac{d-d_{\text {min }}}{v_{a}}
$$

Where:

$v_{i}$ the corrosion rate of time ${ }^{i}$;

$d_{i}$ corrosion of the ${ }^{v_{i}}$ corresponding;

$v_{a}$ the average corrosion rate;

$d$ the remaining wall thickness of the pipe corrosion.

\section{2) Method of Prediction}

\section{a) Determine the minimum allowable thickness}

The minimum allowable thickness $d_{\min }$ is the limit state of pipeline reliability operation, and can be obtained by the formula ( 2-5) or ( 2-6) $d=t-d_{\text {allow }}$.

\section{b) Get the corrosion rate data}

The corrosion rate can be statistical analysis on the basis of the accumulated data. There are two ways to obtain the data, The first method is to make statistics and analysis pipeline repair record over the years, and the data is more accurate. But such data are so rarely that it cannot fully reflect the situation of corrosion in pipeline. The second method is to detect pipeline by smart pigging, and based on the statistics and analysis of previous test data, this method can reflect the overall condition of the pipeline corrosion, is reasonable source of pipeline corrosion rate across the board

\section{APPLICATION OF THE UTILITY FUNCTION IN PIPELINE RELIABILITY.}

\section{A. Establish Utility Function Theory[9]}

The utility refers to the risk, the number of index for decision makers on the consequences of the gain or loss of value preference feeling or reaction, and the utility function is to quantify the decision maker may appear on the decision and state and the possibility of state jointly determine the consequences, is a function of the two, denoted $u=u(a, \theta)$.

Corrosion pipeline segment will have two kinds of state in the operation process: no leakage $a_{1}$ and leakage $a_{2}$ Its probability is $P_{1}, P_{2}$ respectively. When corrosion pipeline leaks, gas leak consequences are generally divided into: steam clouds $\theta_{1}$, jet and pool fires $\theta_{2}$ and explosions $\theta_{3}$, no burning vapor cloud, the probability of no burning vapor cloud, jet fire and explosion were $P_{21} 、 P_{22} 、 P_{23}$ respectively. where, $P_{21}+P_{22}+P_{23}=P_{2}$, . Then in the case of decision $a_{2}$, the utility function of pipe leakage expected according to formula (3-1),

$$
\begin{aligned}
\mathrm{E}_{\mathrm{u}}\left(\mathrm{a}_{2}\right) & =\mathrm{E}\left[\mathrm{u}\left(\mathrm{a}_{2}, \theta\right)\right] \\
& =\mathrm{u}\left(\mathrm{a}_{2}, \theta_{1}\right) p_{21}+\mathrm{u}\left(\mathrm{a}_{2}, \theta_{2}\right) p_{22} \\
& +\mathrm{u}\left(\mathrm{a}_{2}, \theta_{3}\right) p_{23}
\end{aligned}
$$




\section{B. Analyze the consequences of pipeline failure}

1) The remaining life of the failure probability of the pipe section [10]

Experience and theory prove that service life of pipe section obey probability distribution before leak,

$$
P_{s}=e^{-\left(T_{r} / v\right) \alpha}
$$

\section{Where:}

$T_{r} \_$remaining life;

$P_{s} \longrightarrow$ the probability of Service life that reach to ${ }^{T_{r}}$; life";

$v \longrightarrow$ statistical parameters, called "characteristic

$\alpha-$ statistical parameters.

For $T_{r}=v, P_{s}=e^{-1}=0.368$, that is to say, $36.8 \%$ of the pipeline corrosion is not leak that Remaining life has not reached to characterized life. Type (3-2) on both sides of the $\operatorname{logarithm}, \ln T_{r}$ and $\ln \left\{\ln \left[1 / P_{s}\left(T_{r}\right)\right]\right\}$ is a linear relationship.

TABLE I. STATISTICAL PROBABILITY DATA OF THE RESIDUAL LIFE OF A PIPELINE

\begin{tabular}{cccc}
\hline $\begin{array}{c}\text { The } \\
\text { remaining } \\
\text { life }^{T_{r}, \text { a }}\end{array}$ & $\begin{array}{c}\text { Statistical } \\
\text { probability } \\
\text { of life } \\
P_{s}\left(T_{r}\right)\end{array}$ & $\ln T_{r}$ & $\ln \left\{\ln \left[1 / P_{s}\left(T_{r}\right)\right]\right\}$ \\
\hline 1 & 0.94 & 0.00 & -2.78 \\
2 & 0.86 & 0.69 & -1.89 \\
3 & 0.72 & 1.10 & -1.11 \\
4 & 0.50 & 1.39 & -0.37 \\
5 & 0.30 & 1.61 & 0.19 \\
6 & 0.24 & 1.79 & 0.36 \\
7 & 0.14 & 1.95 & 0.68 \\
10 & 0.07 & 2.30 & 0.98 \\
\hline
\end{tabular}

For $x=\ln T_{r}, y=\ln \left\{\ln \left[1 / P_{s}\left(T_{r}\right)\right]\right\}$, Using the least squares $y=a+b x$ curve fitting, we can solve it and get $\alpha=b=1.77$

$a=-2.90$

$v=e^{-a / b}=5.16 v=e^{-a / b}=5.16 \quad$ (A parameter $\alpha$ does not exist).

The results showed that about $63 \%$ of pipeline corrosion perforation leaks, after 5 years and two months. The probability of pipeline residual life is,

$$
P_{f}\left(T_{r}\right)=e^{-\left(T_{r} / 5.16\right)^{1.77}}
$$

Through the prediction of residual life of corrosion pipe, we can get section of the residual life is $T_{r}$, Put $T_{r}$ into (3-3) to get the probability of this section which will continue to use, the probability of leakage is $P_{f}\left(T_{r}\right)=1-P_{s}$ under residual life.

\section{2) Pipeline Failure Loss Value}

Pipeline leakage failure leads to the internal and external loss of the gas pipeline company. value $V_{A}$

a) the pipeline company's internal economic loss

The replacement of serious corrosion of the pipeline in the residual life at $T_{r}$, will cause some damage to the pipeline company, and sunk cost increase in unit length of pipe and reduce the unit length of pipe service turnover. Early replacement of remaining life $T_{r}$ of pipe section will cause a certain degree of sunk costs, the corresponding pipe sunk costs amounted to:

$$
\frac{C}{a_{1}+T_{r}} \times T_{r} \times l
$$

Where:

$C$ - the total unit cost of pipeline construction;

$a_{1} \longrightarrow$ already operational years;

$T_{r}$ the remaining life which has been calculated.

The pipeline which should be replaced can create value for the company in the remaining life $T_{r}$.

$$
\frac{l}{L} \times R_{p r} \times T_{r}
$$

Where:

$l$ - the total length of advance replacement of the pipe's remaining life which is $T_{r}$;

$L \longrightarrow$ the total length of the pipeline;

$R_{p r} \_$pipeline annual average net profit margin;

So expected utility function of such internal losses can be expressed:

$$
V_{A}=\left[\frac{C}{a_{1}+T_{r}} \times T_{r} \times l+\left(\frac{l}{L} \times R_{p r} \times T_{r}\right)\right] \times P_{f}\left(T_{r}\right)
$$

b) the pipeline company's external loss value $V_{B}$ [11]

When the corrosion pipeline fails, the external consequences of the failure considerate three parameters, namely, economic loss, loss of life and environmental damage.

- Economic loss m. Property losses caused by the accident, mainly refers to the value of equipment loss, housing loss, the leakage of natural gas loss.

- Loss of life n. Pipeline leakage accidents caused by casualties, are mainly divided into three cases : the number of staff deaths $N_{1}$ 、 the number of injured personnel $N_{2}$ 、 the number of people with minor injuries $N_{3}$; according to three cases, combined with the local economy that giving the victims' families of financial compensation as a reference .

- Environmental damage h. Natural gas contains toxic and harmful gases, such as $\mathrm{H} 2 \mathrm{~S}$, in the leakage event, damages to the environment, and is very difficult to have a set of calculation method. According to the fine numerical which fits the national specific circumstances on the pipeline companies as a reference.

The above three criteria have no common criteria, multi criteria utility function is drawn by balancing each criteria. Determination of multi criteria utility function can 
equivalently transform multiple standards into a single criterion, which can turn a multi-criteria decision problem into a single- criteria decision problem. Transform multiple criteria utility function into linear combination of single criterion utility function by weighting coefficient, and make decision through a single utility value. Thus, a rule preference degree of a decision maker is not affected by other standards criteria, namely this three criteria are independent of each other, and you can get:

$$
\varepsilon=m+n \times \xi_{1}+h \times \xi_{2}
$$

\section{Where:}

$\xi_{1}$ unit of economic loss of the loss of life;

$$
\begin{aligned}
& N=6000 N_{1}+3000 N_{2}+105 N_{3} \\
& \xi_{1}=\frac{N \times \psi}{n}
\end{aligned}
$$

Among them, $\psi$ Average daily wage casualties, the (3-8) get into (3-9) can be obtained $\xi_{1}$.

$\xi_{2} \longrightarrow$ unit of environmental economic loss caused by the leakage volume.

Set up evaluation index system and calculate leak external pipeline loss expected utility function according to

\begin{tabular}{|c|c|c|}
\hline One & Two & Three \\
\hline \multirow{21}{*}{$\begin{array}{l}1 \text { The } \\
\text { expected value of } \\
\text { the utility } \\
\text { function } \\
E(u)\end{array}$} & & 1.1 .1 \\
\hline & & Economic loss \\
\hline & 1.1 Steam & $m_{1}$ \\
\hline & clouds $\theta_{1}$ & 1.1.2 Loss of \\
\hline & Probability & life $n_{1}$ \\
\hline & $P_{21}$ & 1.1 .3 \\
\hline & & $\begin{array}{c}\text { Environmental } \\
\text { damage } h_{1}\end{array}$ \\
\hline & & 1.2.1 \\
\hline & & Economic loss \\
\hline & 1.2 Jet fires & $m_{2}$ \\
\hline & $\theta_{2}$ & 1.2.2 Loss of \\
\hline & Probability & life $n_{2}$ \\
\hline & $P_{22}$ & 1.2 .3 \\
\hline & & $\begin{array}{c}\text { Environmental } \\
\text { damage } h_{2}\end{array}$ \\
\hline & & 1.3 .1 \\
\hline & & Economic loss \\
\hline & 1.3 & $m_{3}$ \\
\hline & Explosions $\theta_{3}$ & 1.3.2 Loss of \\
\hline & Probability & life $n_{3}$ \\
\hline & $P_{23}$ & 1.3.3 \\
\hline & & $\begin{array}{c}\text { Environmental } \\
\text { damage } h_{3}\end{array}$ \\
\hline
\end{tabular}
the above stated corrosion.

TABLE II. ESTABLISH STATE EVALUATION INDEX SYSTEM
External loss caused by corrosion pipes leakage of expected utility function:

$$
\begin{aligned}
V_{B}= & E_{u}\left(a_{2}\right)=E\left[u\left(a_{2}, \theta\right)\right] \\
= & u\left(a_{2}, \theta_{1}\right) P_{21}+u\left(a_{2}, \theta_{2}\right) P_{22}+u\left(a_{2}, \theta_{3}\right) P_{23} \\
= & \left(m_{1}+n_{1} \times \xi_{1}+h_{1} \times \xi_{2}\right) \times P_{21}+ \\
& \left(m_{2}+n_{2} \times \xi_{1}+h_{2} \times \xi_{2}\right) \times P_{22}+ \\
& \left(\mathrm{m}_{3}+n_{3} \times \xi_{1}+h_{3} \times \xi_{2}\right) \times P_{23}
\end{aligned}
$$

\section{EMPIRICAL ANALYSIS}

A natural gas pipeline has been employed for 40 years, the total length is $210 \mathrm{~km}$, which is divided into 25 pipe valve groups, and the average transmission rate is $600 \times 10^{4} \mathrm{~m}^{3}$ per day approximately within a year. Using API5LX52 to calculate critical defect size, Outside diameter $D=720 \mathrm{~mm}$, Pipe wall thickness $t=10 \mathrm{~mm}$, Yield Strength SMYS=325Mpa, Extreme pressure $P=1.6 \mathrm{Mpa}$, without considering the regional category, by 1.1 to calculate the yield strength, A rectangular pipe defects based on defect formula (2-1) can get the minimum allowable wall thickness. $d_{\min }=\frac{P \times D}{2 \times S M Y S} \times 1.1=1.95$

Therefore, maximum corrosion depth is $d-d_{\min }=8.05 \mathrm{~mm}$.

Using ultrasonic guided wave method and intelligent pigging between every two adjacent segments of the entire valve in the pipeline, various outer surface defect location, size of more precise detection and data will be grouped analysis, select the number of severe pipes corrosion study and classify corrosion size, the degree of similarity of the corroded areas. After classification analysis results in 15 sets of data in the table 3. According to the corrosion rate data and the literature[13]SVM model derived rate of corrosion of the pipeline, and put the results into Equation (2-7) to obtain the remaining life.

TABLE III. STATISTICS SECTION OF THE CORROSION SITUATION BETWEEN THE TWO VALVE GROUP AND THE RESULT OF EVALUATION

\begin{tabular}{ccccc}
\hline $\begin{array}{c}\text { Pipe } \\
\text { segment } \\
\text { number }\end{array}$ & $\begin{array}{c}\text { Remai } \\
\text { ning wall } \\
\text { thickness } \\
\text { mm }\end{array}$ & $\begin{array}{l}\text { Corro } \\
\text { sion wall } \\
\text { thickness } \\
\text { mm }\end{array}$ & $\begin{array}{l}\text { The } \\
\text { average } \\
\text { corrosion } \\
\text { rate } \\
\text { mm/a }\end{array}$ & $\begin{array}{l}\text { Remai } \\
\text { ning life } \\
\text { a }\end{array}$ \\
\hline 1 & 2.72 & 7.28 & 0.228 & 3.38 \\
2 & 2.67 & 7.33 & 0.215 & 3.35 \\
3 & 2.65 & 7.35 & 0.230 & 3.04 \\
4 & 2.62 & 7.38 & 0.226 & 2.96 \\
5 & 2.56 & 7.44 & 0.215 & 2.84 \\
6 & 2.58 & 7.42 & 0.210 & 3.00 \\
7 & 2.60 & 7.40 & 0.211 & 3.08 \\
8 & 2.63 & 7.37 & 0.214 & 3.18 \\
9 & 2.57 & 7.43 & 0.217 & 2.86 \\
10 & 2.66 & 7.34 & 0.220 & 3.23 \\
11 & 2.69 & 7.31 & 0.225 & 3.29 \\
12 & 2.70 & 7.30 & 0.227 & 3.30 \\
13 & 2.59 & 7.41 & 0.217 & 2.95 \\
14 & 2.68 & 7.32 & 0.219 & 3.33 \\
15 & 2.55 & 7.45 & 0.209 & 2.87 \\
\hline
\end{tabular}


The general price of natural gas is 5000 yuan / ton, and per ton of natural gas is equal to $1390 \mathrm{~m}^{3}$. The average of three consecutive years of annual net profits is about 1 billion yuan. Pipeline construction investment cost is $10 \mathrm{billion} / \mathrm{km}$. The selected length between the two valve is $10,000 \mathrm{~m}$. Severe corrosion area (e.g., Fifth, ninth, Fifteenth group detection area) the length of each section together a total length of 1000 meters.

Statistics of various status values is listed as follows:

$$
\begin{aligned}
& m_{1}=1 \times 10^{6}, \quad n_{1}=0, h_{1}=1 ; \\
& m_{2}=2 \times 10^{7}, \quad n_{2}=3, h_{2}=1 ; \\
& m_{3}=7.5 \times 10^{7}, \quad n_{3}=14, h_{3}=3 ; \\
& \xi_{1}=6 \times 10^{6}, \quad \xi_{2}=1 \times 10^{7}
\end{aligned}
$$

The major form of natural gas pipeline leakage accident is toxic gas vapor cloud, jet fire and explosion respectively. American petroleum institute, according to the data provided above all kinds of accident probability is $0.8,0.16$ and 0.04 respectively[13].

$$
\begin{aligned}
V_{A}= & {\left[\frac{C}{a_{1}+T_{r}} \times T_{r} \times l+\left(\frac{l}{L} \times R_{p r} \times T_{r}\right)\right] \times P_{f}\left(T_{r}\right) } \\
= & {\left[\frac{1 \times 10^{8}}{40+T_{r}} \times T_{r} \times 1000+\left(\frac{1}{210} \times 1 \times 10^{10} \times T_{r}\right)\right] } \\
& \times\left(1-P_{s}\right) \\
V_{B}= & E_{u}(a) \\
= & \left(1 \times 10^{6}+1 \times 10^{7}\right) \times 0.8 \\
& +\left(2 \times 10^{7}+3 \times 6 \times 10^{6}+1 \times 10^{7}\right) \times 0.16 \\
& +\left(7.5 \times 10^{7}+14 \times 6 \times 10^{6}+3 \times 10^{7}\right) \times 0.04 \\
= & 2.404 \times 10^{7}
\end{aligned}
$$

For $V_{A}=V_{B}$,put formula (3-3) into (4-1),we can get $T_{r} \approx 0.51, P_{f}\left(T_{r}\right) \approx 0.984$.

When the internal economic loss value $V_{A}$ is greater than the external loss value $V_{B}$, it should replace the corrosion of pipeline in advance in order to prevent the external losses. By above, Fifth, ninth, Fifteenth group detecting corrosion degree similar 1000 meters of pipe should be replace this part of corrosion pipe which residual life is 0.51 .

\section{CONCLUSIONS}

The value of the relevant amount depends on the preferences of the decision-maker in utility function, which has a strong subjectivity, and therefore the different decision-makers using utility function to solve the problem of risk decision may draw the different conclusions for the conclusion. The use of function analysis of effects, is enable to avoid the occurrence of risks to a certain extent, and has a role in early warning, provide reasonable reference for pipeline maintenance 、 repair and Replacement.

This paper needs to be improved :External loss: economic losses only considers the direct economic losses, without considering the secondary economic losses caused by the impact of the spill; The value of life cannot simply measures by the scale of economies, and the poisonous gas may have a long-term damage to the human body. Because gas leak damage has a long-term impact to the environment, using economic measures account simply is unreasonable. Internal lose: just consider the pipeline company's direct costs, without considering the cost of management Therefore, the utility function more conservative and more secure of reliable operation of the pipeline in this paper.

\section{ACKNOWLEDGEMENTS}

The research is supported in part by National Natural Science Foundation support (Foundation Number: 61271278), the name of the fund: The Research on Assessment Technology of Onshore Oil \& Gas pipelines: Supported by the key discipline of Shaanxi Province (E08001). The authors are grateful for the anonymous reviewers who made constructive comments.

\section{REFERENCES}

[1] Yang Zu-pei,Mei Yun-xin , Shuai Jian, Meng Kun-liu. Corrosion of pipeline safety assessment $[\mathrm{J}]$. Gas Storage and Transportation 2003, 22 (5): 1-6.

[2] Wang Yu-mei, Guo Shu-ping. Foreign natural gas pipeline accident analysis [J]. Gas Storage and Transportation 2000, 19 (7): 5-10.

[3] Yang Xiao-heng. Oil and Gas Pipeline Safety Engineering [M]. China Petrochemical Press 2005.

[4] Su Xin, Yang Jun, Yuan Zong-ming, Hu An-xin. Reliability evaluation of pipeline corrosion $[\mathrm{J}]$. Petroleum Engineering Construction 2006, 31 (6): 9-12.

[5] Bai Y, Bai Q. Subsea engineering handbook [M]. 225 Wyman Street, Waltham, MA 02451, USA: Gulf Professional Publishing, 2012.

[6] Bai Qing-dong. The remaining strength of pipeline corrosion [D]; Daqing Petroleum Institute 2006.

[7] Liu Yong-shou, Wang wen, Feng Zhen-zhou, Wang Fu-sheng, Yue Zhu-feng. The remaining strength of corroded pipeline and Reliability Analysis [J]. Strength and Environment 2008, 35 (3): 52-57.

[8] WU Ming-liang,HAO Dian,LIU Jin-kun. Forecast of the Remaining Life of Corrosive Oil and Gas Pipelines Based on GreyMarkov Theory [J]. Pipeline Technique and Equipment.2008, No 5

[9] Zhang Peng, Chen Li-qiong. Optimal risk-based oil and gas pipeline integrity maintenance decision (Two) $[\mathrm{J}]$. Natural Gas Industry.24 (10) : 143-147

[10] Guo Sheng-wu, Yuan Peng-bin. Transmission pipeline integrity testing, evaluation and repair techniques [M]. Petroleum Industry Press.2009, 11, 172-173

[11] Chen Li-qiong. Utility function maintenance decision Optimization in Pipeline Integrity [J]. Gas Storage and Transportation.2004,23 (6) $22-25$

[12] Luo Zheng-shan,, Gao Yang. Corrosion of wet gas gathering pipeline based on gray support vector machines [J]. China Safety Science, 2013, 23 (5)

[13] Luo Zheng-shan,, Gao Yang. Comprehensive quantitative risk assessment of urban natural gas pipeline $[\mathrm{J}]$. Fire Science and Technology.2013.2 